\title{
Impairment of Working Memory, Decision-making, and Executive Function in the First-Degree Relatives of People with Panic Disorder: A Pilot Study
}

\author{
Zhenhe $\mathrm{Zhou}^{1 * \dagger}$ and Dongjie $\mathrm{Ni}^{2+}$ \\ 'Department of Psychiatry, Wuxi Mental Health Center of Nanjing Medical University, Wuxi, China, ${ }^{2}$ Grade 2013 Class 3 , \\ Basic Medicine College of Liaoning Medical University, Jinzhou, China
}

Background: Panic disorder (PD) patients present impairments of working memory, decision-making, and executive function. However, whether the first-degree relatives (FDRs) of people with PD present abnormal characteristics, including clinical and neuropsychological aspects, in comparison to the general population, has not been studied. Investigation and understanding of the abnormal neuropsychological characteristics of the FDRs of people with PD will contribute to the prevention and treatment of PD.

OPEN ACCESS

Edited by:

Amit Anand,

Cleveland Clinic Lerner College of Medicine, United States

Reviewed by:

Federica Sancassiani, Università degli studi di Cagliari, Italy Serafim Carvalho, Hospital de Magalhães Lemos, Portugal

*Correspondence: Zhenhe Zhou

zhouzh@njmu.edu.cn

tThese authors are co-first authors.

Specialty section: This article was submitted to Mood and Anxiety Disorders, a section of the journa

Frontiers in Psychiatry

Received: 15 March 2017 Accepted: 20 October 2017 Published: 03 November 2017

Citation:

Zhou Z and Ni D (2017) Impairment of Working Memory, Decisionmaking, and Executive Function in the First-Degree Relatives of People with Panic Disorder: A Pillot Study.

Front. Psychiatry 8:219.

doi: 10.3389/fpsyt.2017.00219
Objective: The purpose of this paper is to compare the working memory, decisionmaking, and executive function among people with PD, their FDRs, and controls.

Materials and methods: Neuropsychological functions of 30 people with PD, 30 FDRs of people with PD, and 30 controls were measured with a digit span task, lowa Gambling Task (IGT), and Wisconsin Card Sorting Test (WCST).

Results: Perseverative errors, failure to maintain set scores, and number of cards chosen from decks A, B, C, and D were higher for People with PD and their FDRs than those of controls. Furthermore, error rates for these tests were higher for people with $\mathrm{PD}$ than their FDRs. Forward scores and backward scores, percentage of conceptual level responses, the number of categories completed, choices from advantageous minus disadvantageous decks, and mean amount of money earned of people with PD and their FDRs were all lower than those of controls. Scores for these tests were also lower for people with PD than for their FDRs.

Conclusion: People with PD as well as their FDRs present different degrees of impairments of working memory, decision-making, and executive function. Impaired performance on three tasks appears to be associated with the diathesis for PD and may be a valuable indicator of susceptibility for this disorder.

Keywords: panic disorder, first-degree relatives, working memory, decision-making, executive function

\section{INTRODUCTION}

Panic disorder (PD) is a neurosis that presents as frequent panic attacks as well as obvious behavioral changes and leads to many dysfunctions in social function and professional capacity. According to the Diagnostic and Statistical Manual of Mental Disorders, Fifth Edition (DSM-5), $\mathrm{PD}$ is diagnosed as an anxiety disorder based primarily on the occurrence of panic attacks, which 
are persistent and often unexpected. Additionally, at least one attack is followed by the individual fearing that they will have more attacks for at least 1 month. This causes them to change their behavior, which often includes avoiding situations that might induce an attack (1). A common mental disorder, PD affects many people at some point in their lives. During the past three decades, many scholars living in different countries and geographic areas have suggested that the prevalence of PD has been increasing (2). Despite variability in prevalence, many studies have demonstrated consistently that PD is more common among females, in late adolescence or early adulthood, and when combined with depressive disorders and agoraphobia (3-5). For the pathogenesis of $\mathrm{PD}$, many authors suggest that $\mathrm{PD}$ belongs to a disease of polygenic inheritance (6-8). With respect to lifetime prevalence, it has been reported that $\mathrm{PD}$ is 3-21 times higher in the first-degree relatives of people with $\mathrm{PD}$ in comparison to the general population (9). Twin studies showed that PD was heritable and that there is an increased genetic liability in patients with early onset PD $(10,11)$. In a study that investigated temperament in people with $\mathrm{PD}$, their first-degree relatives and healthy individuals, the anxious, depressive, cyclothymiacs, and irritable temperament scores of people with PD were higher than those of healthy individuals, and no differences were reported between the people with PD and their relatives, with the exception of higher anxious temperament scores in people with PD (12). A previous study that investigated the effects of a $35 \%$ $\mathrm{CO}_{2}$ challenge test on healthy, first-degree relatives of people with $\mathrm{PD}$ and on healthy individuals indicated the first-degree relatives were more susceptible to the $35 \% \mathrm{CO}_{2}$ challenge than the healthy individuals (13). Another study investigated anxiety sensitivity among people with PD, their first-degree relatives and controls found that the first-degree relatives were more anxietysensitive than the healthy controls, but less so than the people with PD (14).

Previous studies indicated that people with PD presented impaired information processing and attention as well as impaired interpretation of internal and external stimuli related to the disorder (15). Other studies also reported impairments in short-term memory in people with PD (16). Working memory mainly affects the capacity of brain executive function. A previous study has indicated that the prefrontal cortex is involved in human working memory (17). Additionally, it has been proven that people with PD present a dysfunction of frontolimbic circuits, in particular, orbitofrontal and cingulate cortices (18). Decision-making is an important cognitive function of the human brain. Many studies have reported that people with PD present with impaired decision-making. For example, one study suggested that enhanced cardiac perception may aid intuitive decision-making in healthy individuals, but impair intuitive decision-making in people with PD (19). That study, which used the two-choice prediction task to assess decision-making in people with $\mathrm{PD}$, also showed that people with $\mathrm{PD}$ present abnormality of decision-making function (20). Executive functions are a capacity of the regulation or control for cognitive processes. The prefrontal areas of the frontal lobe are responsible to executive functions (21). A study indicated that people with PD with and without agoraphobia present dysfunctions in episodic memory
(22). Another research study showed that the neurocognitive performance of PD and social phobia patients was lower than that of controls. Furthermore, people with PD exhibited deficits on short-delay free recall and verbal test performance in comparison to controls (23).

Summarily, people with PD present impairments of working memory, decision-making, and executive function. However, whether the first-degree relatives of people with PD present abnormal characteristics, including clinical and neuropsychological aspects, in comparison to the general population is still not reported. Investigation and understanding of abnormal characteristics of the first-degree relatives of people with $\mathrm{PD}$, especially with regard to neuropsychological aspects, will contribute to the prevention and treatment of PD. Clinical psychiatrists and psychologists agree that the digit span task is useful to investigate verbal working memory, that the Iowa Gambling Task (IGT) simulates real-life decision-making, and that the Wisconsin Card Sorting Test (WCST) evaluates prefrontal executive functions. Until now, no studies using Digit Span Task, IGT and WCST in a comparison of working memory, decision-making, and executive functions among people with $\mathrm{PD}$, their first-degree relatives, and controls have been reported.

The purpose of this study was to test whether the first-degree relatives of people with PD presented abnormalities of working memory, decision-making, and executive function compared with controls.

\section{MATERIALS AND METHODS}

\section{Time and Setting}

We performed a cohort study. The experiment was completed in the Department of Psychiatry at Wuxi Mental Health Centre of Nanjing Medical University, China, from May 2013 to January 2015.

\section{Diagnostic Approaches and Participants Study Population PD Group}

Inclusion criteria were as follows: (1) met the DSM-5 criteria for PD; (2) no medication was taken in the past 2 weeks; (3) age range from 18 to 65 years old; (4) were not smokers or alcohol dependent; (5) had never been diagnosed with substance dependence, neurological disorders, or history of head injury. The PD group was recruited from Wuxi Mental Health Centre. Thirty subjects were recruited in the $\mathrm{PD}$ group.

\section{First-Degree Relatives of PD Patient Group}

Participants in the first-degree relatives of people with $\mathrm{PD}$ (FDR-PD) group were selected from the first-degree relatives of patients in the PD group, with no more than one relative chosen for each PD patient, i.e., patient and relative pairs. Inclusion criteria were as follows: (1) no medication received in the past 2 weeks; (3) more than 18 years old; (4) were not smokers or alcohol dependent; (5) had never been diagnosed as substance dependence, neurological disorders or history of head injury. Thirty subjects were recruited as the FDR-PD group. 


\section{Controls}

Thirty persons were selected for the control group, and their age, gender, and education level matched members of the FDR-PD group. Exclusion criteria were as follows: (1) smokers or alcohol dependent; (2) had been diagnosed with substance dependence or a neurological disorder or had a history of head injury; (3) had a family history of mental disorders. The controls were recruited from citizens living in Wuxi city, China through local advertisement.

At the start of the study, a psychiatric resident gathered clinical information about the subjects. The depressive and anxious symptoms of samples were assessed with the Hamilton Depression Scale (HAMD, version of 17 items) and Hamilton Anxiety Scale (HAMA).

All participants were given an informed consent form. All participants provided their own written informed consent to participate in this study and were paid $\$ 31.75$. In this study, a clinician was required to judge whether all people with PD who participated in the study were able to understand the aims and risks of the study and to provide written informed consent. After the assessment, patients were recruited as subjects. The Ethics Committee of Wuxi Mental Health Centre of Nanjing Medical University, China approved the protocol for the research project.

\section{Neuropsychological Tests and Procedures}

The neuropsychological tests were as follows.

Digit Span Task. The Wechsler Adult Intelligence Scale-Revised China (WAIS-RC, computerized version), as described in detail previously (24), was used for measurement of the digit span task. The participant was told to listen because he or she will say a series of numbers and ask the participant to repeat them back in the same order. In this study, two main factors were used for analysis: (1) forward scores; (2) backward scores.

Iowa Gambling Task. The Iowa Gambling Task (IGT, computerized version), as recommended by Shurman et al. and as described in detail previously (25), was used to measure decision-making. Participants are told they may switch from deck to deck as often as they wish and that the overall goal of the task is to maximize profit on a loan of $\$ 2,000$ of "play money." After turning over each card, the subject receives an amount of money. However, on some cards, the subject receives money but also pays a penalty. In our study, choices from advantageous minus disadvantageous decks, mean amount of money earned, and number of cards chosen from decks A, B, C, and D were used for analysis.
Wisconsin CardSorting Test. The WCST (computerizedversion VI), as described in detail previously (24), was used for measurement of executive function. The task entailed matching stimulus cards with one of four category cards in which the stimuli were multidimensional, according to color, shape, and number, with each dimension determining a sorting rule. In our study, five main types of WSCT were used for analysis: (1) the total response errors; (2) percentage of conceptual level responses; (3) perseverative errors; (4) the number of categories completed; (5) failure to maintain set.

\section{Statistical Analysis}

Data were calculated and analyzed using the Statistical Package for the Social Sciences (SPSS, Version 17.0, Inc. Chicago, IL, USA). The male to female sex ratios among members of the PD group, FDR-PD group and controls were analyzed with $\chi^{2}$ tests. A one-way analysis of variance (ANOVA) was used to compare age, years of education among PD group, FDR-PD group, and controls. HAMA and HAMD scores, digit span task scores, IGT scores, and WCST data were analyzed using one-way analysis of co-variance (ANCOVA) by setting age as the covariate. Bonferroni tests were adopted for pairwise comparisons.

\section{RESULTS}

\section{Demographic and Clinical Information of Participants}

The demographic and clinical information of participants is given in the following Table 1.

\section{Comparisons of Digit Span Task Scores among PD, FDR-PD, and Control Groups}

A one-way ANCOVA for forward scores and backward scores revealed a significant main effect of group (PD, FDR-PD, and control groups). Forward and backward scores of the PD group and FDR-PD group were significantly lower than that of controls (for forward scores, $p=0.011$ and 0.013 ; for backward scores, $p=0.010$ and 0.028 ), while the above two factors of the PD group were significantly lower than those of the FDR-PD group (for forward scores, $p=0.025$; for backward scores, $p=0.029$ ) (Table 2).

\section{Comparisons of IGT Scores among PD Group, FDR-PD Group, and Controls}

An one-way ANCOVA for choices from advantageous minus disadvantageous decks, mean amount of money earned, and

TABLE 1 | Demographic and clinical information of participants.

\begin{tabular}{lcccl}
\hline & PD & FDR-PD & Controls & Test statistic \\
\hline Sex ratio (M/F) & $30(13 / 17)$ & $30(15 / 15)$ & $30(15 / 15)$ & $\chi^{2}=6.52, p=0.27, \Phi=0.19$, NS \\
Mean age (SD) & $50(7)$ & $26(6)$ & $26(6)$ & $F(2,87)=22.89, p=0.021$, Cohen's $f=0.50 ;$ PD $>$ FDR-PD and Controls; FDR-PD vs. Controls, NS \\
Age range & $43-56$ & $20-30$ & $20-30$ & - \\
Education (SD) & $9(3)$ & $9(3)$ & $9(3)$ & $F(2,87)=0.19, p=0.73$, Cohen's $f=0.05 ;$ NS \\
HAMA (SD) & $22.3(4.0)$ & $8.2(3.1)$ & $6.3(3.4)$ & $F(2,87)=43.81, p=0.000, \eta^{2}=0.510 ;$ PD $>$ FDR-PD and Controls; FDR-PD vs. Controls, NS \\
HAMD (SD) & $9.5(4.0)$ & $8.0(3.6)$ & $6.9(3.2)$ & $F(2,87)=56.84, p=0.102, \eta^{2}=0.460 ;$ NS
\end{tabular}

$P D$, panic disorder patient group; FDR-PD, the first-degree relatives of people with PD group; M, male; F, female; HAMA, Hamilton Anxiety Scale; HAMD, Hamilton Depression Scale; NS, not significant. 
number of cards chosen from decks A, B, C, and D showed a significant main effect of group (PD, FDR-PD, and control groups). Choices from advantageous minus disadvantageous decks and mean amount of money earned by the PD and FDR-PD groups were significantly lower than those of controls, and the number of cards chosen from decks A, B, C, and D by the PD and FDR-PD groups were significantly higher than those of controls (for choices from advantageous minus disadvantageous decks, $p=0.001$ and 0.019 ; for mean amount of money earned, $p=0.045$ and 0.049 ; for number of cards chosen from $\operatorname{deck} A, p=0.020$ and 0.033 ; for number of cards chosen from deck $B, p=0.043$ and 0.047 ; for number of cards chosen from deck $C, p=0.024$ and 0.028 ; and for number of cards chosen from deck D $p=0.039$ and 0.042).

Choices from advantageous minus disadvantageous decks and mean amount of money earned by the PD group were significantly lower than that of the FDR-PD group ( $p=0.001$ and 0.017 ), and the number of cards chosen from decks A, B, C, and D by the PD group were significantly higher than those of the FDR-PD group ( $p=0.038,0.039,0.044$, and 0.046) (Table 3).

\section{Comparisons of WCST Value among PD, FDR-PD, and Control Groups}

An one-way ANCOVA for response errors, percentage of conceptual level responses, perseverative errors, the number of categories completed, and failure to maintain set showed a significant main effect of group (PD, FDR-PD, and control groups). Total response errors, perseverative errors, and failure to maintain set of the PD group and the FDR-PD group were significantly higher than that of controls, and the number of categories completed and percentage of conceptual level responses of the PD group and FDR-PD group were significantly lower than that of controls (total response errors, $p=0.022$ and 0.017 ; perseverative errors, $p=0.030$ and 0.035 ; failure to maintain set, $p=0.018$ and 0.025 ;

TABLE 2 | Digit Span scores: mean (SD) in PD group $(n=30)$, FDR-PD group $(n=30)$, and control group $(n=30)$.

\begin{tabular}{|c|c|c|c|c|}
\hline Variable & PD & FDR-PD & Controls & Test statistic \\
\hline $\begin{array}{l}\text { Forward } \\
\text { scores }\end{array}$ & $8.5(2.1)$ & $10.0(1.8)$ & $13.3(1.8)$ & $\begin{array}{c}F(2,87)=42.22, \\
p=0.016, \eta^{2}=0.40\end{array}$ \\
\hline $\begin{array}{l}\text { Backward } \\
\text { scores }\end{array}$ & $8.3(2.6)$ & $10.1(2.1)$ & $12.4(2.3)$ & $\begin{array}{c}F(2,87)=38.60 \\
p=0.023, \eta^{2}=0.37\end{array}$ \\
\hline
\end{tabular}

$P D$, panic disorder patient group; FDR-PD, the first-degree relatives of people with $P D$ group. number of categories completed, $p=0.040$ and 0.038 ; and percentage of conceptual level responses, $p=0.010$ and 0.014 ).

Total response errors, perseverative errors, and failure to maintain set for PD group were significantly higher than for the FDR-PD group ( $p=0.026,0.029$, and 0.021$)$, and the number of categories completed and percentage of conceptual level responses of the PD group were significantly lower than that of the FDR-PD group ( $p=0.037$ and 0.043 ) (Table 4).

\section{DISCUSSION}

This study tested working memory, decision-making and executive function among people with PD, their first-degree relatives, and controls. In the present study, working memory was tested with a digit span task, decision-making was measured with the Iowa Gambling Task, and executive function was assessed with the Wisconsin Card Sorting Test. Our results displayed working memory dysfunctions, decision-making impairments, and executive function deficiencies in people with PD and their first-degree relatives compared with controls.

Previous research suggested that working memory involves a system for the temporary storage and manipulation of information, forming a link between controlled action and perception (26). Many studies in animals and functional imaging of humans have identified the parietal cortex, frontal cortex, anterior cingulate, and parts of the basal ganglia involved in working memory (27-30). Our study results showed that two main functions of people with $\mathrm{PD}$ and their first-degree relatives were significantly lower than those of controls by the measurement of the digit span task, and forward scores and backward scores of people with PD were lower compared to their first-degree relatives. These results indicated that people with PD as well as their first-degree relatives all presented working memory dysfunction, with varying degrees of impairment between these two groups.

The Iowa Gambling Task is meant to simulate real-life decision-making processes and reflects the function of the orbitofrontal cortex (OFC) (25). In our study, test scores for choices from advantageous minus disadvantageous decks and mean amount of money earned by people with PD and their first-degree relatives were significantly lower than those of controls, and the number of cards chosen from decks A, B, C, and D by people with $\mathrm{PD}$ and their first-degree relatives were significantly higher than those of controls. Additionally, choices from advantageous minus disadvantageous decks and the mean amount of money earned by people with PD were significantly lower than those

TABLE 3 | IGT scores: mean (SD) in PD group $(n=30)$, FDR-PD group $(n=30)$, and controls $(n=30)$.

\begin{tabular}{|c|c|c|c|c|}
\hline Variable & PD & FDR-PD & Controls & Test statistic \\
\hline Choices from advantageous minus disadvantageous decks & $11.9(10.3)$ & $24.9(12.0)$ & $32.5(14.1)$ & $F(2,87)=41.10, p=0.000, \eta^{2}=0.56$ \\
\hline Mean amount of money earned & $1,913.1(418.2)$ & $2,115.3(449.4)$ & $2,211.5(479.9)$ & $F(2,87)=8.06, p=0.031, \eta^{2}=0.35$ \\
\hline Number of cards chosen from deck $A$ & $19.8(5.0)$ & $18.0(5.4)$ & $15.6(3.2)$ & $F(2,87)=45.08, p=0.039, \eta^{2}=0.40$ \\
\hline Number of cards chosen from deck B & $25.6(6.2)$ & $22.0(3.1)$ & $19.3(5.6)$ & $F(2,87)=50.63, p=0.028, \eta^{2}=0.34$ \\
\hline Number of cards chosen from deck $\mathrm{C}$ & $25.3(6.2)$ & $28.0(5.5)$ & $32.1(6.3)$ & $F(2,87)=54.08, p=0.015, \eta^{2}=0.29$ \\
\hline Number of cards chosen from deck D & $28.1(7.1)$ & $29.0(5.3)$ & $32.7(6.2)$ & $F(2,87)=44.75, p=0.013, \eta^{2}=0.32$ \\
\hline
\end{tabular}

PD: panic disorder patient group. FDR-PD: the first-degree relatives of People with $P D$ group. 
TABLE 4 | WCST data: mean (SD) in PD group ( $n=30)$, FDR-PD group $(n=30)$, and controls $(n=30)$.

\begin{tabular}{|c|c|c|c|c|}
\hline Variable & PD & FDR-PD & Controls & Test statistic \\
\hline Total response errors (\%) & $36.0(11.1)$ & $25.3(12.0)$ & $17.9(18.2)$ & $F(2,87)=33.69, p=0.001, \eta^{2}=0.51$ \\
\hline Conceptual level responses (\%) & $50.1(7.4)$ & $57.9(7.5)$ & $62.5(8.0)$ & $F(2,87)=26.12, p=0.034, \eta^{2}=0.30$ \\
\hline Perseverative errors (\%) & $23.3(11.6)$ & $16.8(12.1)$ & $12.9(10.1)$ & $F(2,87)=53.18, p=0.001, \eta^{2}=0.41$ \\
\hline Number of categories completed & $5.6(2.3)$ & $6.2(3.0)$ & $6.7(3.0)$ & $F(2,87)=58.75, p=0.021, \eta^{2}=0.36$ \\
\hline Failure to maintain set & $0.8(0.5)$ & $0.6(0.7)$ & $0.4(0.2)$ & $F(2,87)=64.19, p=0.015, \eta^{2}=0.28$ \\
\hline
\end{tabular}

$P D$, panic disorder patient group; FDR-PD, the first-degree relatives of people with $P D$ group.

of their first-degree relatives, and the number of cards chosen from decks A, B, C, and D by people with PD were significantly higher than those of their first-degree relatives. Consistent with previous studies $(22,23)$, our results indicated that people with $\mathrm{PD}$ and their first-degree relatives presented different degrees of decision-making impairments.

Because the WCST is sensitive to frontal lobe dysfunctions, it is an assessment of executive process including strategic planning, organized searching, etc. $(21,31)$. Consistent with previous studies $(22,23,32)$, our results showed that some factors of WCST for people with PD and their first-degree relatives were higher than those of controls, and other factors, such as the number of categories completed and the percentage of conceptual level responses, of people with PD and their first-degree relatives were lower than those of controls, which confirmed that people with PD as well as their first-degree relatives presented different degrees of executive dysfunction.

In our study, the first-degree relatives of people with PD exhibited impaired verbal working memory, decision-making, and executive function. These findings cannot be attributed to other factors such as lower education levels, medication effects, etc. Impaired performance on three tasks appears to be associated with the diathesis for PD and may be a valuable indicator of susceptibility for this disorder. Whether performance on the digit span task, IGT, and WCST may be used as an endophenotypic marker for the PD genetic diathesis should be researched in terms of other aspects in the future, such as neuroimaging, neurobiochemistry, and genetics.

\section{REFERENCES}

1. American Psychiatric Association (DSM 5). Diagnostic and Statistical Manual of Mental Disorders: DSM 5. 5th ed. Washington, DC: American Psychiatric Publishing (2013).

2. Sheikh JI, Leskin GA, Klein DF. Gender differences in panic disorder: findings from the national comorbidity survey. Am J Psychiatry (2002) 159(1):55-8. doi:10.1176/appi.ajp.159.1.55

3. Roy-Byrne PP, Katon W, Cowley DS, Russo JE, Cohen E, Michelson E, et al. Panic disorder in primary care: biopsychosocial differences between recognized and unrecognized patients. Gen Hosp Psychiatry (2000) 22(6):405-11. doi:10.1016/S0163-8343(00)00101-8

4. Eaton WW, Anthony JC, Romanoski A, Tien A, Gallo J, Cai G, et al. Onset and recovery from panic disorder in the Baltimore epidemiologic catchment area follow-up. Br J Psychiatry (1998) 173(11):501-7. doi:10.1192/bjp.173.6.501

5. Weissman MM, Bland RC, Canino GJ, Faravelli C, Greenwald S, Hwu HG, et al. The cross-national epidemiology of panic disorder. Arch Gen Psychiatry (1997) 54(4):305-9. doi:10.1001/archpsyc.1997.01830160021003

6. Gratacòs M, Nadal M, Martín-Santos R, Pujana MA, Gago J, Peral B, et al. A polymorphic genomic duplication on human chromosome 15 is a

\section{CONCLUSION}

The first-degree relatives of people with PD presented abnormalities of working memory, decision-making, and executive function compared with controls.

The implication of our study is that understanding the abnormalities of the first-degree relatives of people with PD with respect to neuropsychological functions will contribute to the prevention and treatment of PD. A limitation of this study is that these results are preliminary due to the small sample size. It is necessary to replicate these findings with larger sample sizes in further studies.

\section{ETHICS STATEMENT}

All participants were given a written informed consent and all were paid for $\$ 31.75$, and all participants provided their own written informed consent to participate in this study. The Ethics Committee of Wuxi Mental Health Center of Nanjing Medical University, China approves the protocol for the research project.

\section{AUTHOR CONTRIBUTIONS}

$\mathrm{ZZ}$ and $\mathrm{DN}$ : conceived and designed the experiments, performed the experiments, analyzed the data, contributed reagents/materials/analysis tools, wrote the paper, read and approved the final manuscript.

susceptibility factor for panic and phobic disorders. Cell (2001) 106(3):367-9. doi:10.1016/S0092-8674(01)00447-0

7. Hamilton SP, Slager SL, Heiman GA, Baisre de Leon A, Hodge SE, Knowles J, et al. Evidence for a susceptibility locus for panic disorder near the catechol-o-methyltransferase gene on chromosome 22. Biol Psychiatry (2002) 51(4):591-601. doi:10.1016/S0006-3223(01)01322-1

8. Fyer AJ, Hamilton SP, Durner M, Haghighi F, Heiman GA, Costa R, et al. A third-pass genome scan in panic disorder: evidence for multiple susceptibility loci. Biol Psychiatry (2006) 60(4):388-401. doi:10.1016/j.biopsych.2006. 04.018

9. Vieland VJ, Goodman DW, Chapman T, Fyer AJ. New segregation analysis of panic disorder. Am JMed Genet A (1996) 67(2):147-53. doi:10.1002/ (SICI) 1096-8628(19960409)67:2<147::AID-AJMG4>3.0.CO;2-P

10. Smoller JW, Tsuang MT. Panic and phobic anxiety: defining phenotypes for genetic studies. Am J Psychiatry (1998) 155(9):1152-62. doi:10.1176/ ajp.155.9.1152

11. Bellodi L, Cavallini MC, Bertelli S, Chiapparino D, Riboldi C, Smeraldi E. Morbidity risk for obsessive-compulsive spectrum disorders in first-degree relatives of patients with eating disorders. Am J Psychiatry (2001) 158(4): 563-9. doi:10.1176/appi.ajp.158.4.563 
12. Altinbas G, Altinbas K, Guloksuz SA, Guloksuz S, Aydemir O, Ozgen G. Temperament characteristics in patients with panic disorder and their first-degree relatives. Compr Psychiatry (2015) 60:73-7. doi:10.1016/j. comppsych.2015.03.008

13. Van Beek N, Griez E. Reactivity to a $35 \% \mathrm{CO}_{2}$ challenge in healthy first-degree relatives of patients with panic disorder. Biol Psychiatry (2000) 47(12):830-5. doi:10.1016/S0006-3223(99)00265-6

14. Van BN, Griez E. Anxiety sensitivity in first-degree relatives of patients with panic disorder. Behav Res Ther (2003) 41(8):949-57. doi:10.1016/ S0005-7967(02)00129-8

15. Di Giorgio LM, Velasques BB, Ribeiro P, Nardi AE, de Carvalho MR. Evoked potential in panic disorder patients: a systematic review. CNS Neurol Disord Drug Targets (2015) 14(7):863. doi:10.2174/1871527314666150303164539

16. O'Sullivan K, Newman EF. Neuropsychological impairments in panic disorder: a systematic review. J Affect Disord (2014) 167(167):268-84. doi:10.1016/j. jad.2014.06.024

17. D’Esposito M, Detre JA, Alsop DC, Shin RK, Atlas S, Grossman M. The neural basis of the central executive system of working memory. Nature (1995) 378(6554):279-81. doi:10.1038/378279a0

18. Galderisi S, Mancuso F, Mucci A, Garramone S, Zamboli R, Maj M. Alexithymia and cognitive dysfunctions in patients with panic disorder. Psychother Psychosom (2008) 77(3):182-8. doi:10.1159/000119738

19. Wölk J, Sütterlin S, Koch S, Vögele C, Schulz SM. Enhanced cardiac perception predicts impaired performance in the Iowa gambling task in patients with panic disorder. Brain Behav (2014) 4(2):238-46. doi:10.1002/brb3.206

20. Ludewig S, Paulus MP, Ludewign K, Vollenweider FX. Decision-making strategies by panic disorder subjects are more sensitive to errors. J Affect Disord (2003) 76(1-3):183-9. doi:10.1016/S0165-0327(02)00089-7

21. Alvarez JA, Emory E. Executive function and the frontal lobes: a meta-analytic review. Neuropsychol Rev (2006) 16(1):17-42. doi:10.1007/s11065-006-9002-x

22. Airaksinen E, Larsson M, Forsell Y. Neuropsychological functions in anxiety disorders in population-based samples: evidence of episodic memory dysfunction. J Psychiatr Res (2005) 39(2):207-14. doi:10.1016/j.jpsychires.2004.06.001

23. Asmundson G, Stein M, Larsen D, Walker JR. Neurocognitive function in panic disorder and social phobia patients. Anxiety (1994) 1(5):201-7.

24. Zhou Z, Zhu H, Li C, Wang J. Internet addictive individuals share impulsivity and executive dysfunction with alcohol-dependent patients. Front Behav Neurosci (2014) 8:288. doi:10.3389/fnbeh.2014.00288
25. Shurman B, Horan WP, Nuechterlein KH. Schizophrenia patients demonstrate a distinctive pattern of decision-making impairment on the Iowa gambling task. Schizophr Res (2005) 72(2-3):215-24. doi:10.1016/j.schres.2004. 03.020

26. Baddeley A, Della Sala S, Papagno C, Spinnler H. Dual-task performance in dysexecutive and nondysexecutive patients with a frontal lesion. Neuropsychology (1997) 11(2):187. doi:10.1037/0894-4105.11.2.187

27. Luna B, Minshew NJ, Garver KE, Lazar NA, Thulborn KR, Eddy WF, et al. Neocortical system abnormalities in autism: an fMRI study of spatial working memory. Neurology (2002) 59(6):834-40. doi:10.1212/WNL.59.6.834

28. Chang C, Crottaz-Herbette S, Menon V. Temporal dynamics of basal ganglia response and connectivity during verbal working memory. Neuroimage (2007) 34(3):1253-69. doi:10.1016/j.neuroimage.2006.08.056

29. Mcnab F, Klingberg T. Prefrontal cortex and basal ganglia control access to working memory. Nat Neurosci (2008) 11(1):103-7. doi:10.1038/nn2024

30. Luerding R, Weigand T, Bogdahn U, Schmidtwilcke T. Working memory performance is correlated with local brain morphology in the medial frontal and anterior cingulate cortex in fibromyalgia patients: structural correlates of pain-cognition interaction. Brain (2008) 131(8):3222-31. doi:10.1093/brain/ awn229

31. Miyake A, Friedman NP, Emerson MJ, Witzki AH, Howerter A, Wager TD. The unity and diversity of executive functions and their contributions to complex "frontal lobe" tasks: a latent variable analysis. Cognitive Psychol (2000) 41:49-100. doi:10.1006/cogp.1999.0734

32. Gulcin A, Kursat A, Selin A, Guloksuz S, Aydemir O, Ozgen G. Temperament characteristics in patients with panic disorder and their first-degree relatives. Compr Psychiatry (2015) 60:73-7. doi:10.1016/j.comppsych.2015.03.008

Conflict of Interest Statement: The authors declare that the research was conducted in the absence of any commercial or financial relationships that could be construed as a potential conflict of interest.

Copyright (c) 2017 Zhou and Ni. This is an open-access article distributed under the terms of the Creative Commons Attribution License (CC BY). The use, distribution or reproduction in other forums is permitted, provided the original author(s) or licensor are credited and that the original publication in this journal is cited, in accordance with accepted academic practice. No use, distribution or reproduction is permitted which does not comply with these terms. 